\title{
Investigation of asymmetry of wire-array $Z$ pinches at stagnation using a 4-channel laser diagnostic
}

\author{
V. V. Ivanov, A. A. Anderson \\ University of Nevada, Reno, NV 89557, USA
}

Asymmetry of wire-array Z-pinches at stagnation was investigated using four synchronized laser beams at the wavelength of $266 \mathrm{~nm}$. These beams were spaced at $45^{\circ}$ with respect to each other, allowing a full view of the pinch from four directions. The laser pulse duration was $0.2 \mathrm{~ns}$, with a $<0.1 \mathrm{~ns}$ temporal accuracy between the four channels. Strong asymmetry was found in Z pinches produced by implosion of asymmetrical wire array loads. Anisotropy of the wire-array $Z$ pinch arises due to the asymmetric implosion and development of plasma instabilities. Understanding the three-dimension structure of Z-pinches is important for interpretation of data from $x$-ray and laser diagnostics.

Keywords:

Z-pinch, wire array, implosion, trailing plasma, plasma instability

\section{Introduction}

Dense Z-pinches have been used in different areas of high energy density laboratory plasmas including laboratory astrophysics, atomic physics, and inertial controlled fusion research [1-4]. Plasma instabilities play a crucial role in physics of the $\mathrm{Z}$ pinch. $\mathrm{Z}$ pinches are unstable to different kinds of plasma perturbations [1,5-10] which impact plasma dynamics and limit power and energy of the radiated x-ray pulse. In wire arrays, instabilities arise at the ablation stage, develop during the implosion stage, and seed the $\mathrm{Z}$ pinch at stagnation. $\mathrm{Z}$ pinches are unstable to both the $m=0$ and $m=1$ magnetohydrodynamic (MHD) perturbations at stagnation. MHD necks on the $\mathrm{Z}$ pinch can collapse to micron-scale micropinches and hot spots [6,7]. Fast plasma motion was observed in the $\mathrm{Z}$ pinch at stagnation [8]. The dense pinch is, typically, surrounded by the wide trailing plasma. A three-dimensional (3D) profile of the pinch at the stagnation phase depends on the initial wire array configuration, mass, and wire material. $\mathrm{Z}$ pinches are, typically, studied by $x$-ray and laser diagnostics when one plasma diagnostic collects data from only one azimuthal direction. In this case, data from different plasma diagnostics can be compared if the cylindrical symmetry of the $\mathrm{Z}$ pinch is suggested. However, absorption in plasma may vary in different azimuthal directions if the pinch or trailing plasma are not symmetrical. Therefore, understanding of the spatial profile of the $\mathrm{Z}$ pinch at the stagnation stage may be important for interpretation of data from plasma diagnostics. An asymmetric x-ray yield and power in two orthogonal directions was measured in the planar wire-array $\mathrm{Z}$ pinch [11]. This asymmetry was explained by the initial anisotropy of implosion and radiation absorption in plasma of planar wire-array loads.

Ultraviolet (UV) laser diagnostics at wavelengths of 213-266 nm allow for investigation of the detailed structure of the wire-array dense $\mathrm{Z}$ pinch at the ablation, implosion, and stagnation phases [5,12-15]. The significantly smaller absorption and refraction in dense plasma in the UV range makes it possible to study a spatial profile of the dense part of the $\mathrm{Z}$ pinch hidden by the trailing plasma.

In this paper, spatial profiles of $\mathrm{Z}$ pinches produced by implosion of cylindrical, star, and planar wire arrays were studied at the $1 \mathrm{MA}$ Zebra pulsed power generator by the four-channels 
UV laser diagnostic synchronized with temporal accuracy of $<0.1$ ns. All channels used equivalent beampaths and magnification. Wire-array $\mathrm{Z}$ pinches were investigated at the stagnation stage. Strong azimuthal asymmetry was found in $\mathrm{Z}$ pinches produced by wire array loads with initially asymmetrical configuration. The trailing plasma and plasma jets are sources of the azimuthal asymmetry of $\mathrm{Z}$ pinches. The anisotropic mass distribution can be produced by the asymmetric implosion and development of plasma instabilities at the stagnation stage. Knowing the azimuthal asymmetry of the $\mathrm{Z}$ pinch is important for interpretation of data from $\mathrm{x}$ ray and optical plasma diagnostics.

\section{Experimental setup}

The experiments were carried out with the Zebra pulsed power generator at the Nevada Terawatt facility. The generator produced a 1 MA current pulse with a rising edge of $\sim 80 \mathrm{~ns}$ from

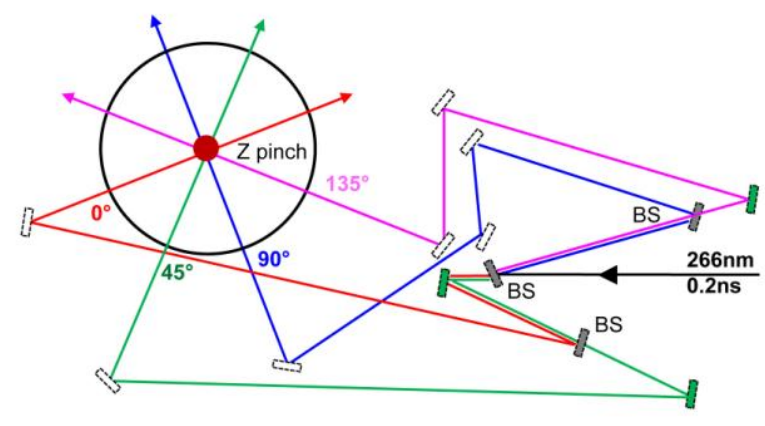

Figure 1 (color online). The experimental setup for the four-channel laser probing of wire-array $\mathrm{Z}$ pinches. $10 \%$ to $90 \%$ of the amplitude. Current was measured by three B-dots placed on the anode plate. The impedance of the transmission line was $1.9 \Omega$. A wire-array load was installed in the return current cage 8 $\mathrm{cm}$ in diameter.

Laser diagnostics of plasma consisted of the four-channel shadowgraphy at the wavelength of $266 \mathrm{~nm}$. A commercial laser Ekspla SL334 generated a laser pulse at the wavelength of $266 \mathrm{~nm}$ with a duration of 0.2 ns and energies of $50 \mathrm{~mJ}$.

UV laser diagnostics have the advantage of less refraction and absorption in dense $\mathrm{Z}$ pinches compared to longer-wavelength probes [5,12]. Refraction angles on plasma gradients depend on the wavelength as $\sim \lambda^{2}$, therefore, refraction is smaller by a factor of 4 at the wavelength of $266 \mathrm{~nm}$ compared to $532 \mathrm{~nm}$. Previous interferometric study at the Zebra generator showed the absence of strong gradients in the trailing plasma [12,22]. A pronounced schlieren effect was not seen in shadowgrams of the $\mathrm{Z}$ pinch. Shadowgraphy presents mostly absorption, so a non-transparent area in the pinch is produced by strong absorption in a dense and large plasma. A contrasting effect on narrow "fingers" of non-imploded material may be linked to the schlieren effect, therefore, a micro-structure of plasma is not discussed here.

Small adsorption in plasma at the wavelength of $266 \mathrm{~nm}$ allows observation of the dense pinch wrapped in the low density trailing plasma [5]. Shadowgraphy is a line integrating diagnostic and the absorption coefficient depends on the plasma electron density, temperature, and ionization stage [12]. Moreover, a shadowgram of the $\mathrm{Z}$ pinch can integrate absorption from plasmas with diverse parameters. $Z$ pinches with the optimal mass for the Zebra generator may have opaque dense cores. However, asymmetry of the pinches can be clearly identified from qualitative shadowgrams.

The input laser beam was split by beamsplitters to four channels with equal energy spaced in $45^{\circ}$ increments as it is shown in Fig. 1. The lengths of channels were equalized with accuracy of $<3 \mathrm{~cm}(<0.1 \mathrm{~ns})$. This accuracy and a short duration of the laser pulse provided instant images of Z-pinch plasma in four azimuthal directions. Four images of plasma were relayed by four beampaths to CCD cameras. Four equivalent three-lens beampaths with the acceptance angle of 
$2.8^{\circ}$ were used to relay images of the pinch to CCDs with the same magnification in all channels. Due to the configuration of "core" diagnostics on the vacuum chamber, the number of mirrors in channels differs but the inter-lens distances were the same. Laser beam profiles were recorded in reference laser shots taken before the main shot. Laser diagnostics occupied eight optical windows on the vacuum chamber of the generator. The $\mathrm{x}$-ray diagnostics included a bolometer, $\mathrm{x}$-ray diodes, and spectroscopy [16]. An x-ray pulse from PCD filtered by $8 \mu \mathrm{m}$ Be foil was used for the timing of the $x$-ray pulse, current pulse, and laser frames.

Wire-array $\mathrm{Z}$ pinches produced by implosion of azimuthally symmetrical cylindrical loads and asymmetrical planar [17] and star [18,19] loads were investigated. All wire arrays were $2 \mathrm{~cm}$ tall and consisted of 8-12 aluminum wires (alloy 5056) 10-15 $\mu \mathrm{m}$ in diameter with a mass of 33$40 \mu \mathrm{g} / \mathrm{cm}$.

\section{Investigation of the spatial structure of $Z$ pinches}

Wire-array $\mathrm{Z}$ pinches were studied at the stagnation stage. Figure 2 shows four shadowgrams of the $\mathrm{Z}$ pinch produced by an $\mathrm{Al}$ 8-wire cylindrical array $16 \mathrm{~mm}$ in diameter.

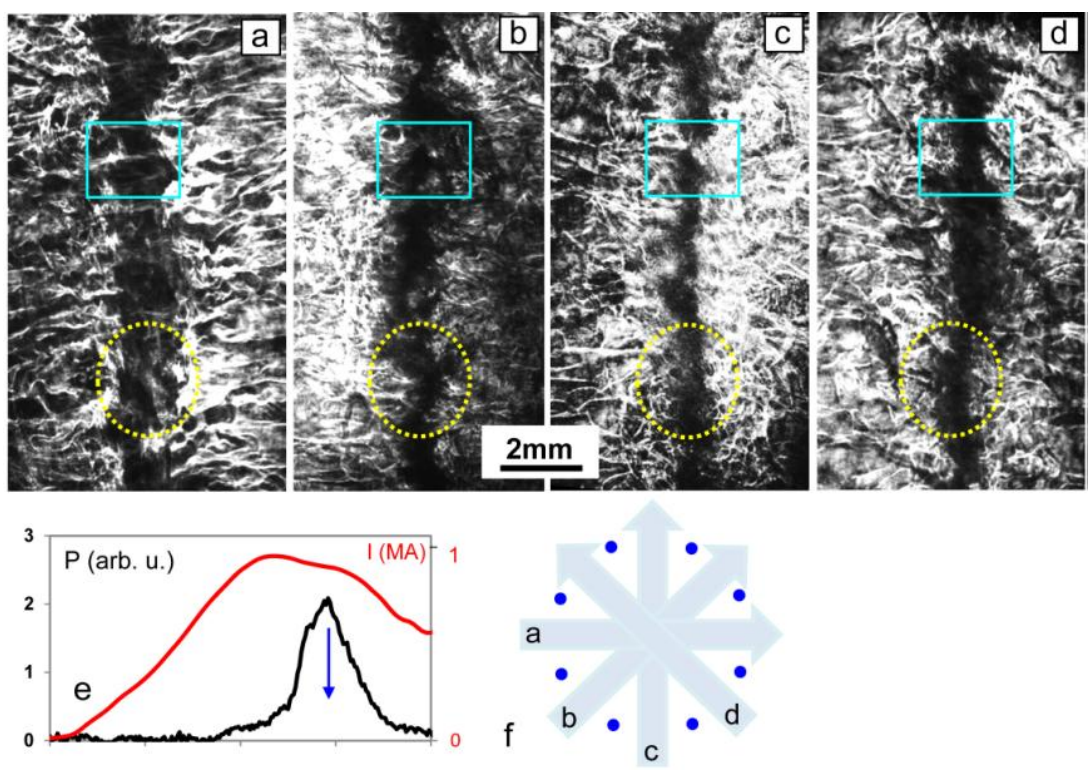

Figure 2 (color online). (a)-(d) Four shadowgrams at $266 \mathrm{~nm}$ from the four-channel laser diagnostics in shot 3458 with $\mathrm{Al}$ cylindrical 8-wire array $16 \mathrm{~mm}$ in diameter. (e) A diagram shows timing of the current pulse (red line), power of the x-ray pulse (black line), and UV frames (arrow). Pictogram (f) shows directions of laser probing for shadowgrams (a) $-(d)$.

Shadowgrams (a) - (d) are taken at the wavelength of $266 \mathrm{~nm}$ from four azimuthal directions of laser probing. Azimuthal directions of laser frames (a) - (d) and configuration of the cylindrical wire array are shown by the pictogram in Fig. 2. A diagram in Fig. 2 (e) shows that laser frames are recorded at the maximum of the $\mathrm{X}$-ray pulse generated by the $\mathrm{Z}$ pinch. In 8-wire arrays all probing directions are placed between the initial positions of wires. This geometry creates the same implosion conditions for the formation of the pinch and trailing plasma in all probing directions. However, the four shadowgrams (a) - (d) depict a difference in the images of the 
pinch. One example is presented in rectangular areas in Fig. 2. The same area of the $\mathrm{Z}$ pinch displays in different directions: (a) a break on the pinch, (b) wide trailing plasma; (c) a micropinch, and (d) a neck on the pinch. Presumably, images vary in different azimuthal directions due to the complicated structure of the trailing plasma and pinch in this location. Images in ovals in Fig. 2 show another example of the variation in shadowgrams in different directions. Images present (a) a loose pinch, (b) a plasma kink with a jet, (c) a neck, and (d) a small kink. A stagnated $\mathrm{Z}$ pinch becomes asymmetrical in areas with strong instability due to the formation of plasma kinks, micropinches, jets and plasma splitting [5-7].

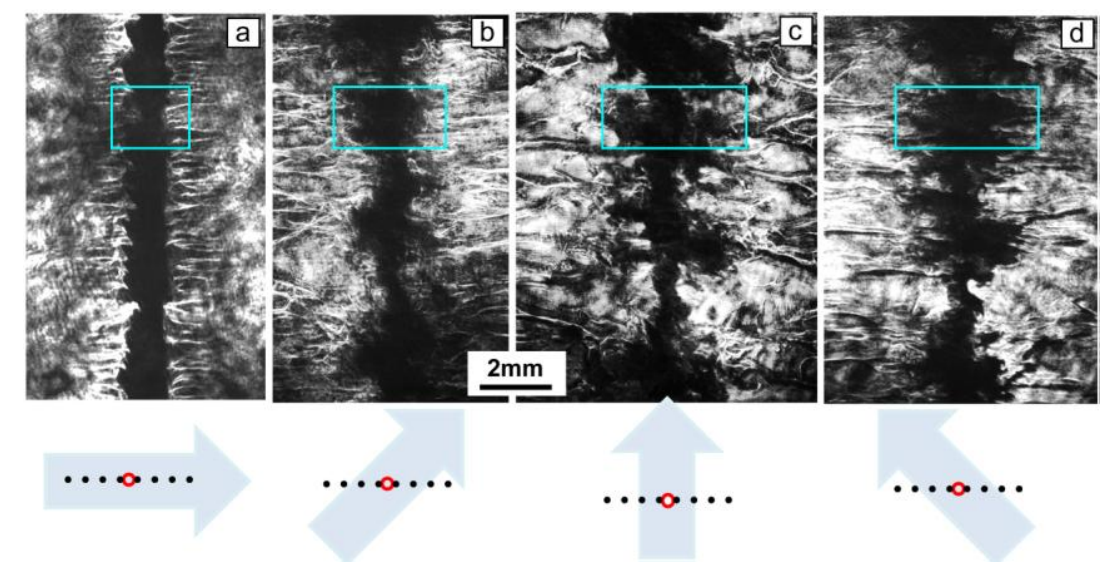

Figure 3 (color online). (a) - (d) Four shadowgrams in shot 3463 with Al planar 8-wire array. Pictograms below shadowgrams show directions of laser probing of the planar wire array.

Figure 3 shows four shadowgrams of the $\mathrm{Z}$ pinch produced by the implosion of the Al planar 8 -wire array with a width of $10 \mathrm{~mm}$ along the line of wires. Planar wire arrays generate high $\mathrm{x}$ ray energy yield and power at the 1 MA Zebra generator $[11,17,20]$. Shadowgrams present a $Z$ pinch at the stagnation stage, $4 \mathrm{~ns}$ after the maximum of the $\mathrm{x}$-ray pulse. Images taken from different directions are very different. Pictograms in Fig. 3 show directions of laser probing. Image (a) taken along the line of wires in the planar array shows a narrow homogeneous pinch. Other images (b) - (d) display a Z-pinch with strong instabilities and wide trailing plasma. This means that trailing plasma stays in the plane of the line of wires during and after the implosion stage. During implosion plasma bubbles pass through the lines of wires and reach the axis [19]. The rest of material implodes later but stays in the plane of the planar array [20]. Wide trailing plasma can absorbs $\mathrm{x}$-ray radiation in one direction and makes asymmetrical radiation of the $\mathrm{Z}$ pinch [11]. This assymetry is also important for laser diagnostics when cylindrical symmetry of plasma is assumed for calculation of plasma density and magnetic fields [21,22]. Rectangles in shadowgrams (a) - (d) present one local area of the pinch taken from four directions. Image (a) shows a neck on the pinch but other images show a large bulge. A $\mathrm{Z}$ pinch of the planar wire array is not cylindrical but exends in the direction of the line of wires.

Figure 4 shows shadowgrams of the $\mathrm{Z}$ pinch produced by the implosion of an Al 3-ray 12wire star array. Star loads are not azimuthally symmetrical and can have 3-6 rays of wires. The implosion in stars occurs in the cascade manner, efficiently snowplows material, and produce plasma columns at the position of the wire closest to the center in every ray $[18,19]$. The cascade implosion produces a more homogeneous $\mathrm{Z}$ pinch with a smaller amount of trailing plasma at stagnation $[10,18]$. 
A star wire-array $\mathrm{Z}$ pinch looks similar in shadowgrams (a) - (d). Images were taken $14 \mathrm{~ns}$ after the maximum of the $\mathrm{x}$-ray pulse when instabilities have already developed. Breaks and bulges are, mainly, located at the same places on the pinch but specific features in plasma can be found. For example, rectangles in shadowgrams (a) - (d) display different configurations of

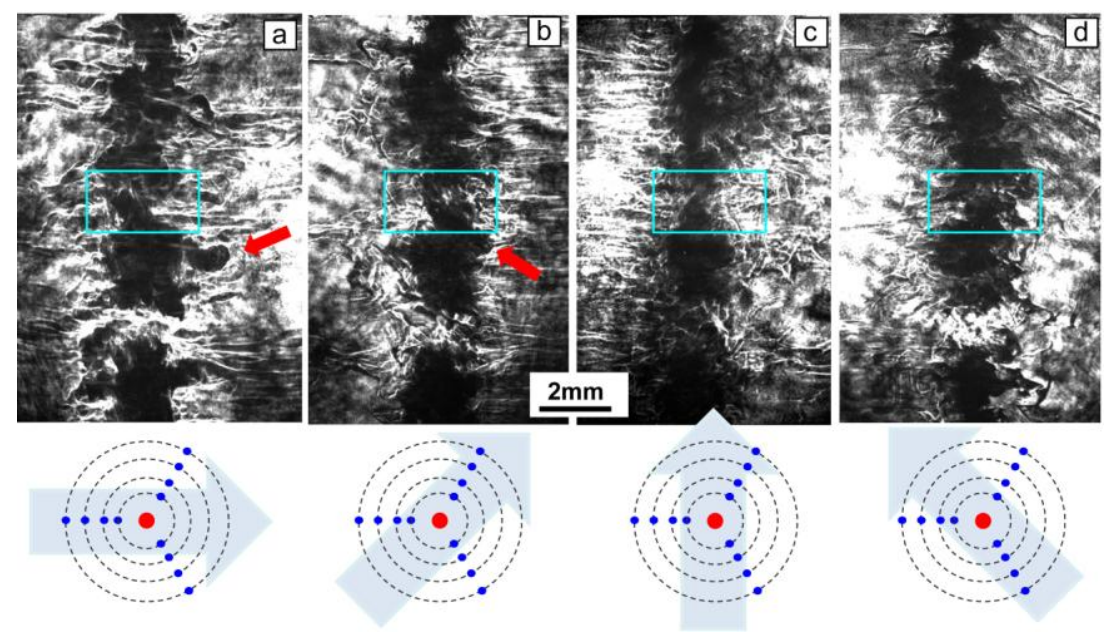

Figure 4 (color online). (a) - (d) Four shadowgrams from shot 3467 with Al 3-ray 12-wire star array. Pictograms below shadowgrams show directions of laser probing of the star wire array.

plasma in the vicinity of the neck. Arrows in Fig. 4 show a plasma jet which is well seen in shadowgram (a) and can be recognized in image (b). A four-channel imaging setup allows the identification of jets, plasma splitting, and other features in the Z-pinch plasma.

\section{Conclusion}

Different types of wire arrays have been used in $\mathrm{Z}$ pinches experiments including different kinds of cylindrical wire arrays [23,24], conical arrays [25], planar arrays [11], star wire arrays [18], and wire arrays for the spherical implosion [26]. Implosions in these wire arrays occur in different ways and produce $\mathrm{Z}$ pinches with different configurations of trailing plasma and pinch. A four-channel UV laser shadowgraphy allows the investigation of the spatial profile of dense plasma near the axis of the $\mathrm{Z}$ pinch which is, typically, hidden in the low-density plasma shell. It was found, that planar arrays produced a $\mathrm{Z}$ pinch with trailing plasma distributed, mostly, in a plane of wires. Cylindrical and star wire array produce more symmetrical plasma distribution at the stagnation stage. However, plasma instabilities form plasma with a complicated asymmetrical structure in some areas on the pinch. Some features of the $\mathrm{Z}$ pinch can be seen only in a specific direction.

The observed 3D features of $\mathrm{Z}$ pinches are in a general agreement with implosion scenarios in wire arrays and development of plasma instabilities. Strong asymmetry in planar wire arrays can be linked to the "planar" implosion along the line of wires [20]. Cascade implosion along 3-4 rays of star arrays, typically, forms a pinch without a kink instability with a smaller amount of trailing material $[18,19]$. Cylindrical wire array $\mathrm{Z}$ pinches demonstrate asymmetry due to plasma instabilities. An axially inhomogeneous implosion seeds instabilities in wire-array $\mathrm{Z}$ pinches. Kink instability is more pronounced in the cylindrical and planar wire arrays [5]. A collapse of 
micro-pinches results in an explosion of plasma and the formation of breaks and jets in wire arrays. 3D MHD modelling of cylindrical and star arrays at the Zebra generator was performed in $[5,10]$.

A spatial profile of Z-pinch plasma can impact interpretation of data from laser and x-ray diagnostics and understanding the physical processes in $\mathrm{Z}$ pinches. For example, asymmetrical plasma can have a higher absorption for x-ray radiation in some directions. Using Abel inversion for calculation of radial profile of the plasma density can lead to additional errors in the asymmetrical distribution of plasma.

Multichannel laser diagnostics can be used for tomographic investigation of the 3D structure of the $\mathrm{Z}$ pinch. Regular wire arrays with masses optimized for $\mathrm{x}$-ray generation were used in our experiments. The dense core of $\mathrm{Z}$ pinches was not transparent for shadowgraphy and interferometry at the wavelength of $266 \mathrm{~nm}$. The electron density up to $(2-3) \times 10^{20} \mathrm{~cm}^{-3}$ can be measured in wire array $\mathrm{Z}$ pinches [12,22] with air wedge interferometry [27] at $266 \mathrm{~nm}$. Light wire arrays are more transparent for UV radiation [12,28] especially at the deep UV wavelength of $213 \mathrm{~nm}$ [14]. Therefore, a full 3D structure of some types of $1 \mathrm{MA} \mathrm{Z}$ pinches can be reconstructed using the deep UV tomography.

\section{Acknowledgments}

The authors acknowledge technical support of experiments from the team of the Nevada Terawatt Facility, UNR. This work was supported by the DOE Grant No. DE-SC0008824 and DOE/NNSA Grant No. DE-NA 0002075.

\section{References}

1. D. D. Ryutov, M. S. Derzon, and M. K. Matzen, Rev. Mod. Phys. 72 (2000) 167.

2. B. A. Remington, R. P. Drake, D. D. Ryutov, Rev. Mod. Phys. 78 (2006) 755.

3. J. E. Bailey, T. Nagayama, G. P. Loisel et al., Nature 517 (2015) 56.

4. M. E. Cuneo, E. M. Waisman, S. V. Lebedev at al., Phys. Rev. E 71, (2005) 046406.

5. V. V. Ivanov, J. P. Chittenden, S. D. Altemara et al., Phys. Rev. Lett. 107 (2011) 165002.

6. V. V. Ivanov, D. Papp, A. A. Anderson et al., Phys. Plasmas 20 (2013) 112703.

7. N. R. Pereira, J. Davis, J. Appl. Phys. 64, R1 (1988).

8. V. V. Ivanov, A. A. Anderson, D. Papp et al., Phys. Rev. E 88, 013108 (2013).

9. S. V. Lebedev, F. N. Beg, S. N. Bland et al., Phys. Plasmas 9 (2002) 2293.

10. V. V. Ivanov, J. P. Chittenden, R. C. Mancini et al., Phys. Rev. E 86 (2012) 046403.

11. V. L. Kantsyrev, A. S. Chuvatin, A. A. Esaulov et al., Phys. Plasmas 20 (2013) 070702.

12. V. V. Ivanov et al., IEEE Trans. Plasma Sci. 42 (2014)1153.

13. S. D. Altemara, D. Papp et al., IEEE Trans. Plasma Sci. 40 (2012) 3378.

14. V. V. Ivanov, A. A. Anderson, I. A. Begishev, Appl. Opt. 55, (2016) 498.

15. A. A. Anderson et al., Phys. Plasmas 22 (2015) 112702

16. V. V. Ivanov, V. I. Sotnikov, J. M. Kindel et al., Phys. Rev. E 79 (2009) 056404.

17. V. L. Kantsyrev, L. I. Rudakov, A. S. Safronova et al., IEEE Trans. Plasma Sci. 34 (2006) 2295.

18. V. V. Ivanov, V. I. Sotnikov et al., Phys. Rev. Lett. 100 (2008) 025004.

19. A. Haboub, et al., IEEE Trans. Plasma Sci. 36 (2008) 1290.

20. V. V. Ivanov, V. I. Sotnikov et al., Phys. Plasmas 14 (2007) 032703.

21. G. S. Sarkisov, A. S. Shikanov, B. Etlitcher, S. Attelan, C. Rouile, JETP Lett. 61 (1995) 485. 
22. V. V. Ivanov et al., Phys. Plasmas 22 (2015) 1100.

23. D. Papp et al., Phys. Plasmas 19, (2012) 092704.

24. S. N. Bland, S. V. Lebedev, J. P. Chittenden et al., Phys. Plasmas 10 (2003) 092710

25. S. V. Lebedev, J. P. Chittenden, F. N. Beg et al., Astrophys. Journal 564 (2002) 113.

26. Y. Zhang, N. Ding, Z. Li et al., Phys. Plasmas 22 (2015) 020703.

27. G. S. Sarkisov, Instrum. Experim. Tech. 39, 727 (1996).

28. A. A. Anderson et al., HEDP 15 (2015) 1. 